\title{
Skin, Hair and Musculoskeletal Complications
}

\author{
Francis Ayuk and Bipin N. Savani
}

\subsection{Complications Involving the Skin and Hair}

\subsubsection{Introduction}

Nearly every recipient of an allo-HSCT will at some stage develop complications involving the skin and hair. These complications can be grouped in drug-related toxicities and allergies, graft-versus-host disease, infections and malignant conditions.

\subsubsection{Drug-Related Toxicities and Allergies}

Drug-related toxicities are most often due to the conditioning regimen, antibiotics or IS agents. Presentation can vary broadly from localised erythema to epidermal necrolysis and StevensJohnson syndrome.

Diagnosis may be difficult because the morphological and chronological presentations of the lesions are generally non-specific. Skin biopsies

\section{F. Ayuk $(\bowtie)$}

Department of Stem Cell Transplantation, University

Medical Center Hamburg-Eppendorf (UKE),

Hamburg, Germany

e-mail: ayuketang@uke.de

\section{B. N. Savani}

Department of Medicine, Vanderbilt University Medical Center, Nashville, TN, USA and histological examination can help improve diagnostic certainty (Paun et al. 2013).

Management requires discontinuation of suspected causative agents, topical treatment with healing ointments, prevention of secondary infections and in severe cases (or when other organs are involved) systemic therapy with corticosteroids and antihistamines.

\subsubsection{Graft-Versus-Host Disease}

The skin is one of the most frequently affected organs in acute and chronic GVHD. Acute GVHD of the skin mainly affects the epidermis of the skin and adjacent oral, anal and genital mucosa. Chronic GVHD may affect all layers of the skin including the epidermis, dermis and subcutaneous tissue and may also manifest as skin dyspigmentation, sweat impairment, alopecia and thinning of scalp hair, hair loss in other areas (e.g. eye brows) and nail dystrophy. Chronic GVHD with sclerosis of the subcutaneous tissue including fasciae, joints and the musculoskeletal system can severely impact patients' quality of life. Sclerodermal lesions of the thorax or abdomen may impair breathing, lesions adjacent to joints may impair movement, and genital lesions may cause phimosis, vaginal scarring and narrowing of the introitus that may cause dyspareunia and even complete obliteration of the vaginal tract. 
In the early "inflammatory" phase of GVHD, patients often present with oedema and discomfort which later progress to fibrosis and joint contractures. Regular survey of range of motion by patients and physicians may enable early detection of reversible lesions. Regular assessment (e.g. via questionnaire or oral interview) during routine clinical visits may encourage physicians and patients to address issues involving the genital tract, thereby enabling early detection and treatment.

General treatment and management of GVHD discussed in Chaps. 43 and 44. In patients with chronic GVHD of the skin and subcutaneous tissues, treatment is best initiated in the early phase prior to development of fibrosis and contractures, which otherwise may require protracted immunosuppression and other measures, sometimes over several years. Physiotherapy including deep myofascial massage and stretching exercises is essential to restore or maintain range of motion. In patients with genital involvement, topical treatment with immunosuppressive agents and hormones and use of vaginal dilators should be initiated early to prevent or reduce the degree of irreversible fibrosis and avoid the need for surgical intervention.

\subsubsection{Infectious Complications Involving the Skin}

Infection-associated skin lesions are often due to viruses. Unexplained fever and rash are more frequent in patients with HHV6 viremia compared to controls (Betts et al. 2011). Due to lack of effective prophylaxis, HHV6-related complications occur during both early and late transplant phases, while shingles (varicella zoster) are mostly seen beyond 6 months after transplant and mainly after discontinuation of prophylactic aciclovir. Other infectious conditions of the skin include fungal infections (mainly due to dermatophytes and, less frequently, Aspergillus or Mucor species) and bacterial infections. Management of infections mostly consists of systemic antiviral, antifungal or antibiotic treatment.

\subsubsection{Malignant Complications of the Skin}

Post transplant malignant conditions of the skin include skin cancer (basal cell carcinoma, squamous cell carcinoma and melanoma) and relapse of underlying malignant disease. BCC and SCC are much more common and have a better prognosis than melanoma. The incidence of melanoma has been reported to be higher after allo-HSCT with standardised incidence ratios ranging from 1.4 to 8.3 (Inamoto et al. 2015). Secondary cancers, risk factors and management are discussed in detail in Chap. 47.

Patients should be counselled to perform selfexamination of the skin and adjacent mucosa, use adequate sun protection and avoid excessive sun exposure. Country-specific general population recommendations for screening for cancer should be adapted and modified taking increased risk of HSCT survivors into consideration.

\subsection{Musculoskeletal Complications}

\subsubsection{Introduction}

Complications involving the muscles include myopathies, myositis and cramps. Musculoskeletal complications are reported in 35\% of long-term survivors 10 years after allogeneic transplantation (Syrjala et al. 2005).

\subsubsection{Myopathy}

The most frequent causes of myopathy early after transplant are corticosteroid therapy and inactivity. Patients report muscles weakness with no pain, and laboratory investigations show normal creatinine kinase. The proximal lower limb muscles, particularly the quadriceps muscles are most severely affected. The main risk factors include increasing dose and duration of corticosteroid therapy, older patient age and the extent and 
duration of inactivity, particularly when intensive care is required. Patients should receive physiotherapy as soon as corticosteroid therapy is initiated and be advised to exercise on their own. Systemic corticosteroids should be tapered or avoided when possible.

\subsubsection{Myasthenia Gravis}

Though rare $(<1 \%)$, it has been reported after allogeneic stem cell transplantation, mostly in the context of chronic GVHD. Patients present with fatigable weakness during or after tapering of immunosuppression (Grauer et al. 2010). Diagnosis includes detection of antibodies against acetylcholine in blood. Treatment consists of cholinesterase inhibitors and IS therapies for chronic GVHD.

\subsubsection{Muscle Cramps}

Muscle cramps are painful and often visible contractions lasting up to $30 \mathrm{~min}$. Though rarely reported, they appear to be frequent in patients with chronic GVHD (Filipovich et al. 2005); an association with chronic IS may also be possible. Magnesium deficiency and side effects of medications (e.g. ganciclovir, valganciclovir) should always be ruled out. If magnesium replacement and discontinuation of suspected causative drugs do not bring relief, treatment with, e.g., quinine or antiepileptic drugs may be considered.

\subsubsection{Myositis}

Myositis has been reported in up to $3 \%$ of patients after allo-HSCT. Though frequently associated with other symptoms of chronic GVHD, it can also be the sole manifestation of GVHD (Openshaw et al. 2009). Patients often present with pressure-sensitive muscle pain and increased blood creatine kinase. Management is within GVHD treatment (Couriel et al. 2002).

\subsubsection{Complications Involving the Bones and Joints}

The most frequent complications involving the bones and joints are chronic GVHD, avascular osteonecrosis and bone loss (osteopenia/osteoporosis). Chronic GVHD of the joints is discussed in Chap. 44.

\subsubsection{Osteoporosis/Osteopenia}

Osteopenia (defined as a T-score between -1 and -2.5 ) and osteoporosis (defined as a T-score less than -2.5 ) have been reported in about $25-50 \%$ of patients after allo-HSCT and up to $60 \%$ in patients with severe chronic GVHD (Pirsl et al. 2016). Risk factors include protracted IS, older patient age, higher cumulative corticosteroid dose (Schulte and Beelen 2004; Savani et al. 2007; Stern et al. 2001; Yao et al. 2008; Petropoulou et al. 2010; Abou-Mourad et al. 2010) and lower body weight, malnutrition, physical inactivity, female gender, higher average $\mathrm{NIH}$ organ score as well as higher platelet counts in patients with severe chronic GVHD (Pirsl et al. 2016).

In accordance with these risk factors, decrease in bone mineral density occurs most rapidly within the first year after transplant. If osteopenia or osteoporosis is diagnosed, endocrine causes like hyperthyroidism, hyperparathyroidism and hypogonadism need to be ruled out. Screening using dual energy X-ray absorptiometry (DEXA) is recommended 1 year after transplant and repeat measurements in patients with recognised defects (Majhail et al. 2012).

Measures to prevent bone loss include vitamin D supplementation in regions with high prevalence of vitamin D deficiency, adequate calcium intake preferable through diet and regular weightbearing physical exercise. Beyond the above preventive measures, specific treatment of patients with severe osteopenia or osteoporosis include bisphosphonates or denosumab. Hormone replacement therapy should be considered in patients with hypogonadism. Patients should also be counselled to modify negative lifestyle factors 
(e.g. cease smoking) and take measures to prevent falls (e.g. physical exercise including balance training, correct visual disorders).

\subsubsection{Avascular Necrosis}

Avascular necrosis (AVN) has been reported in up to $19 \%$ of adult patients and up to $29 \%$ of patients younger than 20 years (Torii et al. 2001; Patel et al. 2008). Risk factors include GVHD, steroid therapy, microvascular changes due to GVHD and/or its therapy, younger age at transplant and TBI (Socié et al. 1997; French et al. 2008; Patel et al. 2008; Campbell et al. 2009; Jagasia et al. 2010). Patients usually present with joint pain, restricted to one or two affected joints. Though most joints can be affected, the hips are by far most frequently involved, bilateral in the majority of cases.

Screening for AVN is not recommended; however high index of suspicion and prompt MRI are necessary in symptomatic patients with risk factors, to enable early detection and intervention.

Pain relief and maintenance or restoration of patient mobility are the main aims or treatment. Discontinuation of corticosteroid and other IST should be considered where possible. Drug therapy is limited to pain relief. The role of nonsurgical causative therapies, such as bisphosphonates, statins and prostacyclin analogues, is still unclear. Pressure relief by means of surgical core decompression may relief pain and slow down progression in early stages, whereby additional autologous bone marrow grafting further improves long-term outcome (Hernigou et al. 2018). In patients with late-stage disease with femoral head collapse, jointpreserving strategies are not effective, and total hip arthroplasty is the recommended long-term treatment.

\section{References}

Abou-Mourad YR, Lau BC, Barnett MJ, et al. Long-term outcome after Allo-SCT: close follow-up on a large cohort treated with myeloablative regimens. Bone Marrow Transplant. 2010;45:295-302.

Betts BC, Young JA, Ustun C, et al. Human herpesvirus 6 infection after hematopoietic cell transplantation: is routine surveillance necessary? Biol Blood Marrow Transplant. 2011;17:1562-8.

Campbell S, Sun CL, Kurian S, et al. Predictors of avascular necrosis of bone in long-term survivors of hematopoietic cell transplantation. Cancer. 2009; 115:4127-35.

Couriel DR, Beguelin GZ, Giralt S, et al. Chronic graftversus-host disease manifesting as polymyositis: an uncommon presentation. Bone Marrow Transplant. 2002;30:543-6.

Filipovich AH, Weisdorf D, Pavletic S, et al. National Institutes of Health consensus development project on criteria for clinical trials in chronic graft-versus-host disease: I. diagnosis and staging working group report. Biol Blood Marrow Transplant. 2005;11:945-56.

French D, Hamilton LH, Mattano LA, et al. A PAI-1 (Serpine1) polymorphism predicts osteonecrosis in children with acute lymphoblastic leukemia: a report from the Children's Oncology Group. Blood. 2008;111:4496-9.

Grauer O, Wolff D, Bertz H, et al. Neurological manifestations of chronic graft-versus-host disease after allogeneic haematopoietic stem cell transplantation: report from the Consensus Conference on Clinical Practice in chronic graft-versus-host disease. Brain. 2010;133:2852-65.

Hernigou P, Dubory A, Homma Y, et al. Cell therapy versus simultaneous contralateral decompression in symptomatic corticosteroid osteonecrosis: a thirty year follow-up prospective randomized study of one hundred and twenty five adult patients. Int Orthop. 2018. https://doi.org/10.1007/s00264-018-3941-8.

Inamoto Y, Shah NN, Savani BN, et al. Secondary solid cancer screening following hematopoietic cell transplantation. Bone Marrow Transplant. 2015;50:1013-23.

Jagasia S, Misfeldt A, Griffith M, Jagasia M. Age and total body irradiation in addition to corticosteroid dose are important risk factors for avascular necrosis of the bone. Biol Blood Marrow Transplant. 2010;16:1750-1.

Majhail NS, Rizzo JD, Lee SJ, et al. Recommended screening and preventive practices for long-term survivors after hematopoietic cell transplantation. Bone Marrow Transplant. 2012;47:337-41.

Openshaw H, Vogelsang GB, Pavletic SZ. Neurological manifestations of chronic graft versus host disease, chronic graft versus host disease. New York: Cambridge University Press; 2009. p. 243-51.

Patel B, Richards SM, Rowe JM, et al. High incidence of avascular necrosis in adolescents with acute lymphoblastic leukemia: a UKALL XII analysis. Leukemia. 2008;22:308-12.

Paun O, Phillips T, Fu P, Novoa RA, Honda KS, Lu KQ, Lazarus HM. Cutaneous complications in hematopoietic cell transplant recipients: impact of biopsy on patient management. Biol Blood Marrow Transplant. 2013;19(8):1204-9.

Petropoulou AD, Porcher R, Herr AL, et al. Prospective assessment of bone turnover and clinical bone diseases 
after allogeneic hematopoietic stem-cell transplantation. Transplantation. 2010;89:1354-61.

Pirsl F, Curtis LM, Steinberg SM, et al. Characterization and risk factor analysis of osteoporosis in a large cohort of patients with chronic graft-versus-host disease. Biol Blood Marrow Transplant. 2016;22:1517-24.

Savani BN, Donohue T, Kozanas E, et al. Increased risk of bone loss without fracture risk in longterm survivors after allogeneic stem cell transplantation. Biol Blood Marrow Transplant. 2007;13:517-20.

Schulte CM, Beelen DW. Bone loss following hematopoietic stem cell transplantation: a long-term follow-up. Blood. 2004;103:3635-43.

Socié G, Cahn JY, Carmelo J, et al. Avascular necrosis of bone after allogeneic bone marrow transplantation: analysis of risk factors for 4388 patients by the Société Francaise de Greffe de Moelle (SFGM). Br J Haematol. 1997;97:865-70.
Stern JM, Sullivan KM, Ott SM, et al. Bone density loss after allogeneic hematopoietic stem cell transplantation: a prospective study. Biol Blood Marrow Transplant. 2001;7:257-64.

Syrjala KL, Langer SL, Abrams JR, et al. Late effects of hematopoietic cell transplantation among 10-year adult survivors compared with case-matched controls. J Clin Oncol. 2005;23:6596-606.

Torii Y, Hasegawa Y, Kubo T, et al. Osteonecrosis of the femoral head after allogeneic bone marrow transplantation. Clin Orthop Relat Res. 2001;382:124-32.

Yao S, Mc Carthy PL, Dunford LM, et al. High prevalence of early-onset osteopenia/osteoporosis after allogeneic stem cell transplantation and improvement after bisphosphonate therapy. Bone Marrow Transplant. 2008;41:393-8.

Open Access This chapter is licensed under the terms of the Creative Commons Attribution 4.0 International License (http://creativecommons.org/licenses/by/4.0/), which permits use, sharing, adaptation, distribution and reproduction in any medium or format, as long as you give appropriate credit to the original author(s) and the source, provide a link to the Creative Commons license and indicate if changes were made.

The images or other third party material in this chapter are included in the chapter's Creative Commons license, unless indicated otherwise in a credit line to the material. If material is not included in the chapter's Creative Commons license and your intended use is not permitted by statutory regulation or exceeds the permitted use, you will need to obtain permission directly from the copyright holder. 